\title{
Development of novel tunable dual-band negative index metamaterial using open stub- loaded stepped-impedance resonator
}

\begin{abstract}
This study reports on tunable planar metamaterial design that is capable to achieve dual-band negative index of refraction responses operating in microwave regime. Its distinctive characteristic is the usage of tuning open stub-loaded stepped-impedance resonators. Parameters retrieval algorithm, and full-wave simulation of prism-shaped structure were carried out to validate the negative refraction characteristics of metamaterial structure. The results predict its prospect as a very promising alternative to the conventional ones, which is compatibly applicable on various potential microwave devices especially when dual-band function is required. In addition to that, its design flexibility offers a various frequency bands at any possible choice, which is alterable together with any design parameters changes.
\end{abstract}

Keyword: Metamaterials; Dual-band; Microwave devices; Refractive index 